\title{
ANÁLISIS DE LA DONACIÓN RENAL EFECTIVA POR MUERTE ENCEFÁLICA EN UN HOSPITAL DE NIVEL 2 DE LA COMUNIDAD DE MADRID EN UN PERÍODO DE 10 AÑOS
}

\author{
Daniel Santos Arrontes, Manuel Pablo Martín Muñoz, Jesús Isidoro García González, Pedro \\ Galdos Anuncibay', Fernando Gimeno Albo y Pedro Paniagua Andrés.
}

Servicio de Urología. Hospital de Móstoles. Madrid. Unidad de Cuidados Intensivos'. Coordinación de Trasplantes. Hospital de Móstoles. Madrid. España.

\begin{abstract}
Resumen.- OBJETIVOS: Las características epidemiológicas del donante en muerte encefálica se han modificado a lo largo del tiempo sin un incremento significativo en la tasa de explantes efectuados.
\end{abstract}

Evaluar si las características epidemiológicas (sexo, edad y causa de exitus) del donante en muerte encefálica se han modificado a lo largo del tiempo. Evaluar si la tasa de explantes efectivos se ajustan a un estándar de calidad.

MÉTODOS: En este estudio retrospectivo se incluyeron todos los pacientes con donación renal (una o dos unidades renales) en el período comprendido entre el 1 de enero de 1995 y el 31 de diciembre de 2005, en un hospital de Nivel 2 con Unidad de Cuidados Intensivos, pero sin Neurocirugía y sin ser centro de referencia de

Daniel Santos Arrontes

Servicio de Urología

Hospital de Móstoles

Calle Rio Júcar s/n

28935 Móstoles. Madrid. (España).

dsantosa@gmail.com

Trabajo recibido: 5 de junio 2007. politraumatismos severos, en un área sanitaria de la Comunidad de Madrid (Móstoles, 200.000 habitantes). Se evaluó la causa de éxitus, la edad, serología para VHB o VHC, así como la donación conjunta de otros órganos (hígado, páncreas, intestino, corazón, pulmón y tejidos). Además se evaluarán las diferencias entre dos periodos de tiempo consecutivos: 1995-1999 y 2000-2005. Para el análisis estadístico se aplicará el Test $T$ para muestras independientes, considerándose como significativo un error alfa inferior a 0.05, mediante el Software SPSS v13.0 (Chicago, Illinois, USA) para Windows. Para el ajuste de la tasa de explantes se utilizará como indicador de calidad un mínimo de un $0,2 \%$ del total de exitus intrahospitalario.

RESULTADOS: Durante todo el periodo a estudio hubo 4314 exitus en todo el ámbito hospitalario. Se realizaron 46 extracciones renales con una edad media de 58.1 años (error estándar de la media -eem- de 14.25). En el primer periodo (1995-1999) se produjeron 20 donaciones con una edad media de 52.8 años (eem 12.13) y en el segundo periodo (2000-2005) se produjeron 26 donaciones con una edad media de 62.23 (eem 14.49) años. No obstante no existieron diferencias estadísticamente significativas entre ambos grupos ( $p=0.273)$. En total 8 pacientes fueron VHB positivos (17.4\%) y $4(8.7 \%$ ) VHC positivo. En el $80.4 \%$ de los pacientes la causa de muerte encefálica fue una hemorragia cerebral (37 pacientes), el $15.2 \%$ por encefalopatía hipóxica (7 pacientes), un paciente falleció por embolia grasa tras accidente de tráfico y otra por púrpura trombótica. No existieron diferencias en cuanto a la causa de exitus entre ambos periodos (p>0.05). Se realizó extracción hepática en el 74 \% de los pacientes, cardiaca y pulmonar en el 6,5\%, pancreática en el $4,3 \%$ y de tejido en el $50 \%$. La tasa de donación fue de un $1,05 \%$. 
CONCLUSIONES: 1. Existe una tendencia al alza en la edad del donante en muerte encefálica, aunque sin significación estadística, probablemente debido al tamaño muestral. 2. Las tasas de detección de donantes en muerte encefálica se encuentra por encima de los estándares de calidad. No obstante, se deberían establecer programas formativos a nivel hospitalario para la detección de estos pacientes y mejorar los resultados obtenidos.

Palabras clave: Trasplante. Epidemiología.

Summary.- OBJECTIVES: The epidemiological characteristics of brain-dead donors have changed with time without a significant increase in the rate of explants performed. The objective of this article is to evaluate if the epidemiological characteristics /sex, age, and death cause) have changed with time, and To evaluate if the rate of effective explants is adjusted to a quality standard.

METHODS: All patients who became renal donors lone or two renal units) between January 1 st 1995 and December 37 st 2005 in a level II hospital with intensive care unit but without neurosurgery, which is not a reference center for severe polytrauma patients, within a health-care area of the Autonomic Community of Madrid (Mostoles, 200.000 inhabitants), were included in this retrospective study. Cause of death, age, HBV and $\mathrm{HCV}$ serologies, as well as multiorgan donation lliver, pancreas, bowel, heart, lungs, and tissues). AdditionaIly, the differences between two consecutive periods of time will be analyzed: 1995-1999 and 2000-2005. SPSS v 13.0 software (Chicano, Illinois, USA) was used for the statistical analysis; the T test for independent samples was applied, considering an alpha error inferior to 0.05 for significance. A minimum of $0.2 \%$ of the total number of hospital deaths was considered as quality indicator for the adjustment of donation rate.

RESULTS: Over the whole study period there were 4314 deaths in the hospital. Forty-six renal harvestings were performed with a mean donor age of $58.1 \mathrm{yr}$. Imean standard error (SE) 14.25). In the first study period (1995-1999) there were 20 donations with a donor mean age of $52.8 \mathrm{yr}$ (SE 12.13), and there were 26 donations in the second period (2000-2005) with a mean age of 62.23 yr. (SE 14.49). Nevertheless, there were no statistically significant differences between both groups ( $p=2.273$ ). A total of eight patients were $\mathrm{HBV}$ positive (17.4\%) and four (8.7\%) HCV positive. In $80.4 \%$ of the patients the cause of brain death was brain hemorrhage (37 patients), 15.2\% hypoxic encephalopathy (7 patients), one patient died due to fat embolism after motor vehicle accident, and another one from thrombotic purpura. There were no differences in death cause between both periods ( $p>2.05$ ). Hepatic extraction was performed in $74 \%$ of the patients, heart and lung in $6.5 \%$, pancreas in $4.3 \%$, and tissues in $50 \%$. Donation rate was $1.05 \%$.

CONCLUSIONS: 1. There is a trend to higher braindead-donor age, although it is not statistically significant, probably due to sample size. 2. The rate of brain-dead donor detection is over quality standards. Nevertheless, training programs to detect these patients and improve results should be established at the hospital level.

Keywords: Transplantation. Epidemiology.

\section{INTRODUCCIÓN}

El fallecimiento de un individuo en téminos biológicos no es un proceso instantáneo sino un proceso evolutivo durante el cual van extinguiéndose las distintas funciones de los órganos corporales, finalizando cuando todas las células del individuo han cesado irreversiblemente su función. En la actualidad el fracaso completo e irreversible de las funciones del sistema nervioso central (SNC) se considerá la frontera entre la vida y la muerte del ser humano, ya que el fracaso neurológico completo se acompaña irremediablemente del cese de las funciones cardíacas y repiratoria, aunque dado el desarrollo de técnicas de soporte (como la ventilación mecánica) este cese puede diferirse (1).

Entendemos por tanto como muerte encefálica global (MEG) al cese irreversible de las funciones neurológicas de los hemisferios cerebrales y del tronco del encéfalo. Por las características de estos pacientes constituyen la base fundamental para la donación de órganos y tejidos.

Diversos estudios realizados muestran que entre un 1 y un $5 \%$ de los pacientes que fallecen en un hospital lo hacen en situación de muerte encefálica (este valor puede alcanzar el $16 \%$ si tenemos en cuenta sólo las Unidades de Cuidados Intensivos). Lamentablemente no todas las MEG son detectadas, por lo que se consideran efectivas unas cifras de donación aquellas que son superiores al $20 \%$ de las MEG (2-5). Del mismo modo, las características epidemiológicas del donante se han modificado a lo largo del tiempo con el fin de incrementar la tasa de trasplantes.

Nuestros objetivos son evaluar si las características epidemiológicas del donante en MEG se han modificado a lo largo del tiempo, así como determinar si la tasa de explantes efectivos se ajustan a éste estándar de calidad. 


\section{MATERIAL Y MÉTODOS}

En este estudio retrospectivo se incluyeron todos los pacientes con donación renal (una o dos unidades renales) en el período comprendido entre el 1 de Enero de 1995 y el 31 de Diciembre de 2005, en un hospital de Nivel 2 con Unidad de Cuidados Intensivos, pero sin Neurocirugía, y que no es centro de referencia para politraumatismos severos, en un área sanitaria de la Comunidad de Madrid (Móstoles, 200.000 habitantes). Se evaluó la causa de éxitus, la edad, serología para hepatitis B (VHB) - $C(\mathrm{VHC})$, así como la donación conjunta de otros órganos (hígado, páncreas, intestino, corazón, pulmón y tejidos). Además se evaluarán las diferencias epidemiológicas entre dos periodos de tiempo consecutivos: un primer período que comprendería desde el año 1995 hasta el año 1999 inclusive, y un segundo período que abarcaría desde el año 2000 al 2005. Para el análisis estadístico se aplicará el test T para muestras independientes, considerándose como significativo un error alfa inferior a 0.05, mediante el Software SPSS v13.0 (Chicago, Illinois, USA) para Windows. Para el ajuste de la tasa de explantes se utilizará como indicador de calidad un mínimo de un $0,2 \%$ del total de muertes encefálicas detectadas en el área a estudio.

\section{RESULTADOS}

Durante todo el periodo a estudio hubo 4314 exitus en todo el ámbito hospitalario. Se reali-

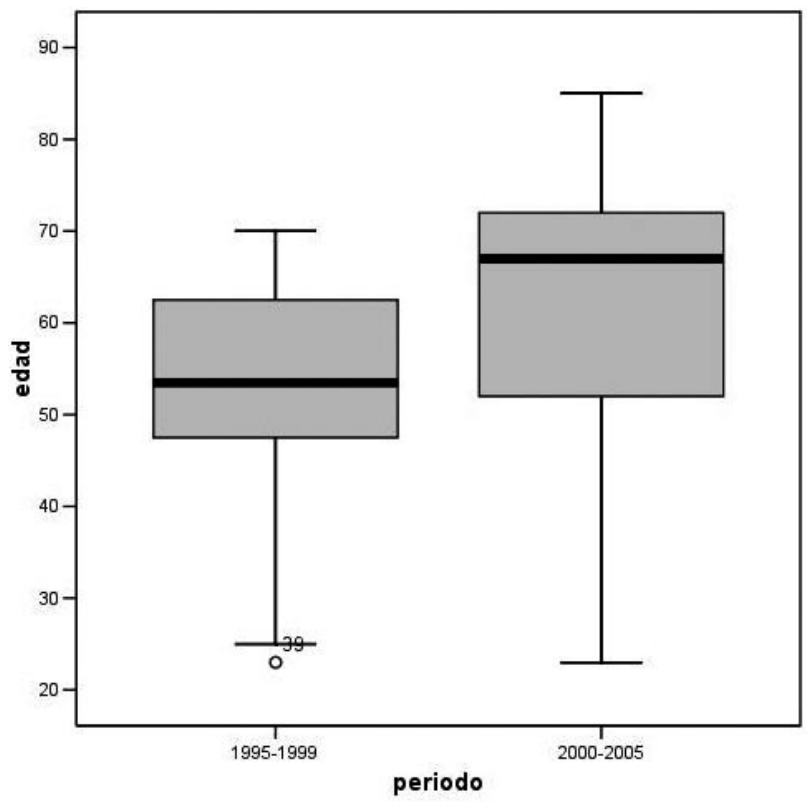

FIGURA 1. Representación gráfica de la edad del donante para cada uno de los períodos. zaron 46 extracciones renales con una edad media de 58.1 años (error estándar de la media -eem- de 14.25). La distribución por sexos fue de 32 varones $(69,5 \%)$ y 14 mujeres $(30,4 \%)$. En el primer periodo (1995-1999) se produjeron 20 donaciones con una edad media de 52.8 años (eem 12.13) y en el segundo periodo (2000-2005) se produjeron 26 donaciones con una edad media de 62.23 (eem 14.49) años. No obstante no existieron diferencias estadísticamente significativas entre ambos grupos $(p=0.273$ ) (Figura 1). En total 8 pacientes fueron VHB positivos $(17.4 \%)$ y $4(8.7 \%)$ VHC positivo. En el $80.4 \%$ de los pacientes la causa de muerte encefálica fue una hemorragia cerebral (37 pacientes), el 19,6\% por encefalopatía hipóxica $(9$ pacientes, incluyendo un paciente que falleció por embolia grasa tras accidente de tráfico y otra por púrpura trombótica). No existieron diferencias en cuanto a la causa de exitus entre ambos periodos $(p>0.05)$. Se realizó extracción hepática en el $74 \%$ de los pacientes, cardiaca y pulmonar en el $6.5 \%$, pancreática en el $4,3 \%$ y de tejido en el $50 \%$. La tasa de donación fue de un $1,05 \%$.

\section{DISCUSIÓN}

En la Comunidad de Madrid 15.000.000 habitantes) existe un registro de MEG desde el año 1991. Entre 1991 y 2002 se registraron 3558 fallecidos en MEG, con una media de 297 MEG/año (268-318), lo que supone aproximadamente 60 MEG por millón de habitantes y año. Se detectaron un $2 \%$ de las MEG en el ámbito hospitalario, lo que ha supuesto 149 donantes reales $1 \%$ de los éxitus hospitalarios) (Figura 2). En nuestro medio los resultados obtenidos por tanto se encuentran por encima del estándar de calidad, con una tasa de donación de un $1,05 \%$ de los exitus hospitalarios $(6,7)$.

En España la mayoría de los donantes son varones $(63 \%)$, siendo las causas más frecuentes de exitus el accidente cerebrovascular (ACVA- 61,5\% de las donaciones), el traumatismo craneoencefálico (TEC- $16,1 \%$ ) y la encefalopatía hipóxico-isquémica $(22,4 \%)$. Además existe una tendencia a la reducción de la mortalidad por TCE y a un incremento en la mortalidad por ACVA que se mantiene durante los últimos años. En nuestro medio, dadas las características del centro, la mayor parte de las donaciones tienen su origen en un ACVA, y está situación ha permanecido inmutable a lo largo de todo el período. Por el contrario, la edad media del donante fue ligeramente superior a la global para España $(58,1 \pm$ 14,25 versus $52,5 \pm 17,4$ ) debido probablemente a las propias características demográficas de la población (8). Del mismo modo al no tratarse de un centro 


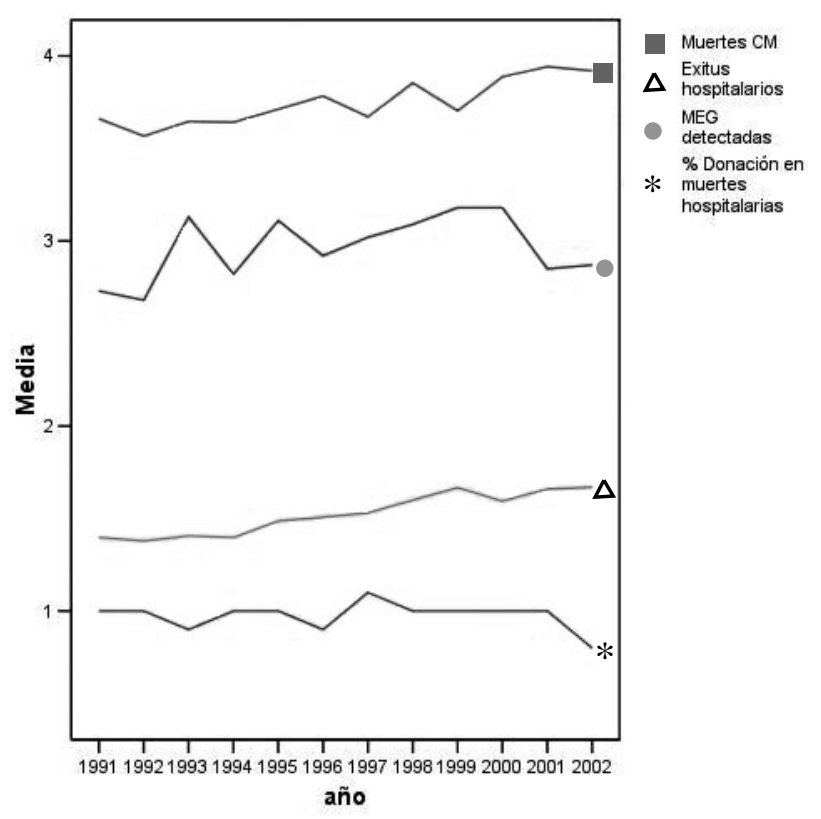

FIGURA 2. Representación gráfica de la incidencia absoluta de muerte en la Comunidad de madrid (CM, línea azul), exitus hospitalarios (línea roja), muertes encefálicas globales detectadas en el ámbito hospitalario y porcentaje de donantes por MEG. Para el ajuste de escalas el número de muertos y el número de éxitus por año están divididos por 10.000, y el número de MEG entre 100

trasplantador no existen casos con donante vivo que si están incluidos para el cálculo global de la edad del donante para España, pudiendo desplazar el valor de la media a valores ligeramente inferiores. Bien es cierto que el donante vivo está muy poco desarrollado en España, constituyendo únicamente un $4 \%$ de la actividad trasplantadora.

En cuanto a la distribución por sexos, esta sigue una distribución similar a la descrita con un predominio de varones que constituyen más del $60 \%$ de los donantes. Sin embargo llama la atención la proporción de pacientes con serologías positivas para $\mathrm{VHB}$ y $\mathrm{VHC}(17,4$ y $8,7 \%$ respectivamente) que son muy superiores a los valores registrados para todo el país. Según la Organización Nacional de Trasplante (ONT), en el año 2005, el 0,8 \% de los donantes presentaron positividad para el VHB, oscilando entre un 0,5 y un $1 \%$ en los últimos 10 años. la serología positiva para VHC en el año 2005 fue de 2,5 $\%$, oscilando los últimos años entre un 1,3 y un 2,6 $\%$. Estas diferencias no pueden ser justificadas salvo que exista una mayor prevalencia de infecciones por virus VHB y VHC en el área sanitaria a estudio. Sin embargo este hecho no es posible de constatar.
Otra tendencia que puede apreciarse es un incremento en la edad de donación durante el periodo a estudio en todas las series (8). En la nuestra no existen diferencias significativas entre los dos períodos aleatorios establecidos pero probablemente esto sea debido al pequeño tamaño muestral.

\section{CONCLUSIÓN}

1. Existe una tendencia al alza en la edad del donante en muerte encefálica, aunque sin significación estadística, probablemente debido al tamaño muestral.

2. Las tasas de detección de donantes en muerte encefálica se encuentra por encima de los estándares de calidad. No obstante, se deberían establecer programas formativos a nivel hospitalario para la detección de estos pacientes y mejorar los resultados obtenidos.

\section{BIBLIOGRAFIA y LECTURAS RECOMENDADAS ( ${ }^{*}$ lectura de interés $y^{* *}$ lectura fundamental)}

1. ESCALANTE COBO, J.L.: "En Manual de Coordinación de trasplantes". Editado por TPM, Les Heures, Universitat de Barcelona y Fundació Bosch i Gimpera. Barcelona, 2005.

*2. NAVARRO, A.: "Brain death epidemiology: The Madrid Study". Trasplant Proc; 28: 103, 1996.

3. ESPINEL, E.; DEULOFEU, R.; SABATER, R. y cols.: " The capacity for organ generation of hospitals in Catalonia, Spain: A multicentre Study". Trasplant Proc; 21:1419, 1989.

4. ARANZABAL, J.; PERDIGO, L.: "Organ procurement organization in the Basque Autonomous Community: Present achievements and future prospects". Trasplant Proc; 22:335, 1990.

5. MIRANDA, B.; FERNÁNDEZLUCAS, M.; MATESANZ, R.: "The potential organ donor pool: international figures". Trasplant Proc; 29:1604, 1997.

6. NAVARRO, A.; ESCALANTE, J.L. y cols.: "Detection of donors and organs procurements in the Autonomous Community of Madrid". Intensive Care Med; 18: 83, 1992.

7. NAVARRO, A.; ESCALANTE, J.L.; ANDRÉS, A. y cols.: "Donor detection and organ procurement in the Madrid region". Transplant Proc, 25: 3130, 1993.

**8. GARRIDO-CANTARERO, G.; MATESANZ, R.: "Epidemiología de la donación y el trasplante en España". Enfer Infecc Microbiol Clin; 25:54, 2007. 